\title{
Investigation of the Stress in MOS Structures with Micro-Raman Scattering
}

\author{
P. Borowicz ${ }^{a, b}$, L. Borowicz ${ }^{a}$ AND D. BrzezińsKA ${ }^{a}$ \\ ${ }^{a}$ Institute of Electron Technology, al. Lotników 32/46, 02-668 Warsaw, Poland \\ ${ }^{b}$ Institute of Physical Chemistry PAS, Kasprzaka 44/52, 01-224 Warsaw, Poland
}

\begin{abstract}
Two kinds of samples were investigated with micro-Raman spectroscopy. MOS structures having poly-silicon gates with metallic contact were made of $\mathrm{Al}$ with addition of $1 \% \mathrm{Cu}$. The other type of the sample was Si wafer subjected to surface oxidation. The position of the sample was changed along $z$-axis in order to maximize the Raman signal coming from different layers of the structure. Two MOS structures were studied, both prepared in the same process, and after that only one was thermally-treated at temperature $400^{\circ} \mathrm{C}$. The spectra coming from different layers of the structure were analyzed numerically using Lorentzian profile. The authors want to point out that the measurements of the Raman signal performed through the metallic contact of the poly-silicon gate gave anomalously large signal coming from silicon structure. This effect was observed only for thermally threated sample. This suggest that in the case of thermally-treated structure the signal observed through the metallic contact was magnified by surface-enhanced Raman scattering phenomenon.
\end{abstract}

PACS numbers: 78.30.Fs, 73.40.Qv, 32.70.Jz, 78.30.-j

\section{Introduction}

Raman scattering is a very suitable method for investigation of the mechanical stress in MOS structures [1, 2]. The typical structure under study consist of: silicon substrate, dielectric layer, gate made of metal or poly-silicon with metallic contact. The cross-section through the structure studied in this work is shown in Fig. 1. The

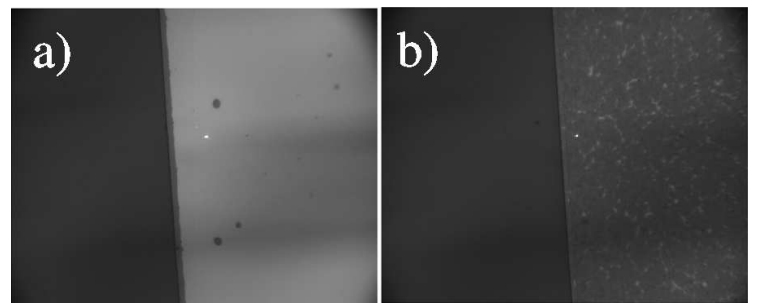

Fig. 1. Microscopic images of MOS structures: (a) as-deposited; (b) annealed by thermal treatment at $400^{\circ} \mathrm{C}$.

intensity of Raman scattering is very low in comparison with the intensity of laser beam inducing the scattering. Since the penetration depth of visible light in the case of $\mathrm{Si}$ is about $0.5 \mu \mathrm{m}$, the Raman signal coming from silicone substrate is relatively large. There are also two conditions that make interpretation of the scattering coming from the Si substrate relatively easy:

1. the scattering is detected as narrow, well-defined line because the material has generally crystal structure;

2. the mathematical model of the dependence between Raman scattering and mechanical stress is worked out [3-6].
In the case of dielectric $\left(\mathrm{SiO}_{2}\right.$ layer $)$ the situation is not so comfortable. First: the efficiency of Raman scattering of $\mathrm{SiO}_{2}$ is small in comparison with that of $\mathrm{Si}$. Second: the amount of material that can be irradiated is small due to thickness of dielectric layer which is generally of the order of $10 \mathrm{~nm}$. Third: $\mathrm{SiO}_{2}$ is an amorphous material, so the laser light is scattered by many similar structures having slightly different geometries. The Raman bands of $\mathrm{SiO}_{2}$ are not sharp and well-defined like $\mathrm{Si}$ lines but broad with maxima that are not well defined. Also the signal-to-noise ratio in the case of $\mathrm{SiO}_{2}$ Raman spectra is much smaller in comparison with scattering from $\mathrm{Si}$ substrate.

\section{Experimental}

All samples, MOS structures and Si wafers, were manufactured in Division of Silicon Microsystem and Nanostructure Technology.

The Raman scattering was measured with MonoVista Raman microscope (Spectroscopy and Imaging GmbH, Germany). As a fundamental the visible line of $\mathrm{Ar}^{+}$laser $488 \mathrm{~nm}$ (INOVA 90C FREED) was used. The power of the laser beam on the sample was equal to $\approx 1 \mathrm{~mW}$.

\section{Results and discussion}

In Fig. 1 is presented the microscopic image of MOS structures with poly-silicon gate covered by metallic contact ( $\mathrm{Al}$ doped with $1 \% \mathrm{Cu}$ ). Part (a) presents the structure without thermal treatment, part (b) after annealing at $400^{\circ} \mathrm{C}$. Bright part of each image shows gate covered with metallic contact, the dark one - the Si substrate covered with $\mathrm{SiO}_{2}$ layer. The bright spot in the center of 
each photo shows the focused laser beam. The surface of the contact of as-deposited samples shows smooth structure. The surface of metallic contact of annealed structures is rough.

One of the most interesting information about the MOS structures is this one that can be obtained from the area covered with metallic contact. This is however not easy, because the metallic contact has very strong absorption, so incident and scattered light are efficiently absorbed by metallic layer. So it is very important to find the optimal condition for the Raman measurements of the part of MOS structures that are placed below the metallic layer. In Fig. 2 there are presented Raman spectra measured as $z$-scans of the samples presented in Fig. 1. Part (a) presents spectra measured for as-deposited structure, part (b) — for annealed one. Both measurements were done through the metallic contact a few $\mu \mathrm{m}$ from the edge. The main conclusions coming from this measurement can be expressed as follows:

1. no signal was observed in the case of as-deposited structure;

2. the intensity of Raman scattering coming through the metallic contact from annealed structure was almost the same as for the measurements performed on the area not covered by metallic layer.

Since the intensity of scattering obtained through metallic contact doped with $\mathrm{Cu}$ was unexpectedly large, the reference measurements were performed. Raman scattering was measured for the annealed sample having aluminum gate without addition of $\mathrm{Cu}$. The signal observed through the metallic gate was in this case few orders of magnitude smaller.

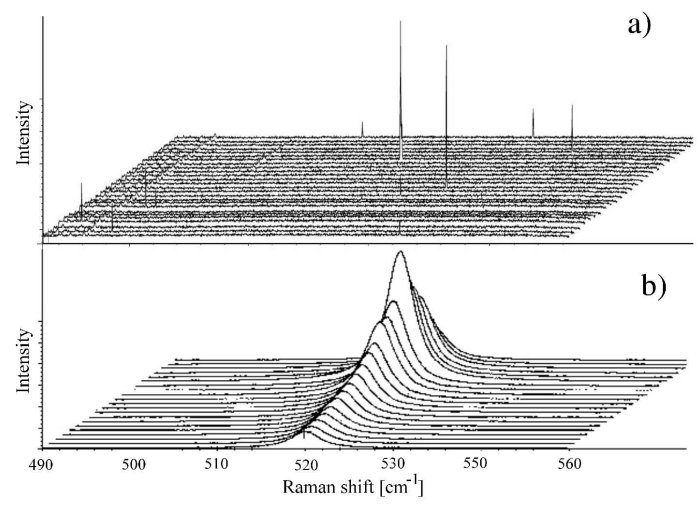

Fig. 2. Raman spectra of $z$-scan: (a) measured for as-deposited sample, (b) measured for annealed sample. Following frames correspond to the following positions in $z$-direction

Authors focused their attention at two aspects of relationship between Raman scattering and mechanical stress in MOS structures.

The first one is the interpretation of the spectroscopic data in terms of back scattering. In this treatment vibra- tion of the sample parallel to the optical axis are not detected. In the case of optical microscopes large aperture of objectives, the component of electric field of incident light parallel to optical axis is non-negligible. The same is valid for the scattered light: the component parallel to optical axis of the objective is non-negligible. The other aspect is the distribution of stress along $z$-axis.

In order to get more detailed insight into the details of the interaction between $\mathrm{Si}$ substrate and $\mathrm{SiO}_{2}$ layer the $z$-scan of the "simplified MOS" structures were performed. "Simplified MOS" structure means silicon wafer with $\mathrm{SiO}_{2}$ layer without metallic or poly-silicon gate. This kind of structure preserves the main feature of the sample, it means the mechanical stress created during the process of surface oxidation due to differences in molar volumes of $\mathrm{Si}$ and $\mathrm{SiO}_{2}$ and removes others sources of mechanical stress namely gates. Since $\mathrm{Si}$ is non-transparent medium (the penetration depth for visible light equals to about $0.5 \mu \mathrm{m}$ ) the $z$-scan results in the change of the thickness of the layer from which the scattered light is detected (effective penetration depth). This change is caused by different density of laser light at different distances from focal point of the microscope objective. The price to pay is the loss of space resolution due to increase of the spot of the laser beam when the $\mathrm{Si} / \mathrm{SiO}_{2}$ interface is moved out of the focus of the microscope objective. However, in the case of "simplified MOS" structures the spatial resolution is of minor importance.

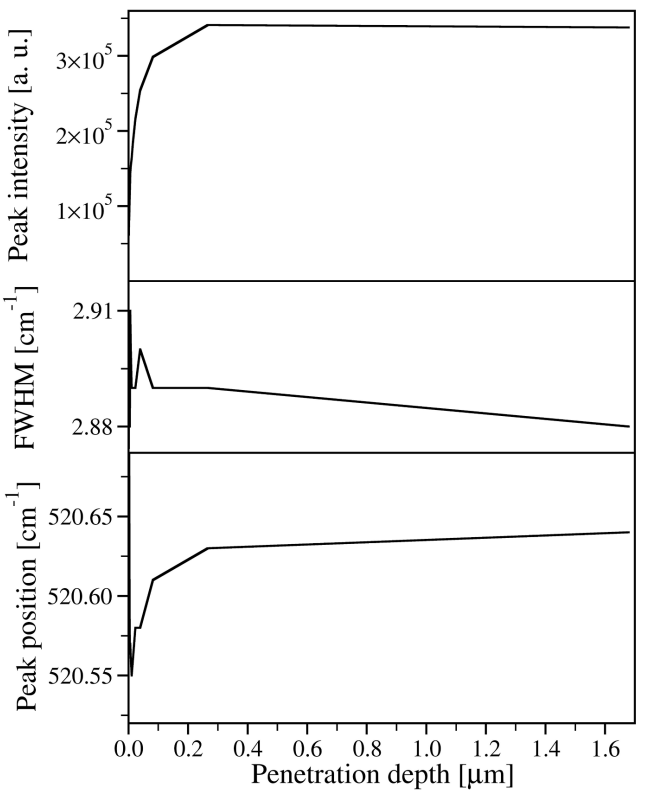

Fig. 3. Analysis of $z$-scan Raman spectra with single Lorentzian profile (silicon peak). The following frames present (starting from bottom): maximum of Lorentzian profile, FWHM of the profile and peak intensity.

The Raman peak coming from silicon substrate was analyzed with function consisting of linear part (to reproduce background) and one or two Lorentzian profiles. 
In Fig. 3 are presented (starting from the bottom frame): the position of the maximum of Lorentzian profile, the halfwidth (FWHM) of the profile and the intensity of Raman peak. All data are presented as a function of effective penetration depth. The effective penetration depth was calculated from the imaginary part of the refractive coefficient of Si at $488 \mathrm{~nm}$, dimension of the focused laser beam (for $488 \mathrm{~nm}$ the diameter of the focal spot equals to $1 \mu \mathrm{m})$ and the aperture of microscopic objective. It was assumed that for the interface placed in the focal plane of the objective the effective penetration depth should be equal to $3 \mu \mathrm{m}$. It is clearly seen that the maximum of Raman peak moves to smaller values of Raman shift (Rs) with the decrease of penetration depth. The analysis of autocorrelation function shows that the scattering is not reproduced well with single Lorentzian profile. The fitting procedure gives better results if two Lorentzian profiles are used. The results of fitting procedure are presented in Fig. 4. The following frames (starting from bottom) present: position of maxima of Lorentzian profiles, their FWHM's and the intensity of peak 1. The intensity ratio of peaks 2 to 1 is between 0.2 and 0.3 . The course of autocorrelation function shows that the model containing two Lorentzian profiles much better reproduces the Raman spectra than single Lorentzian profile.

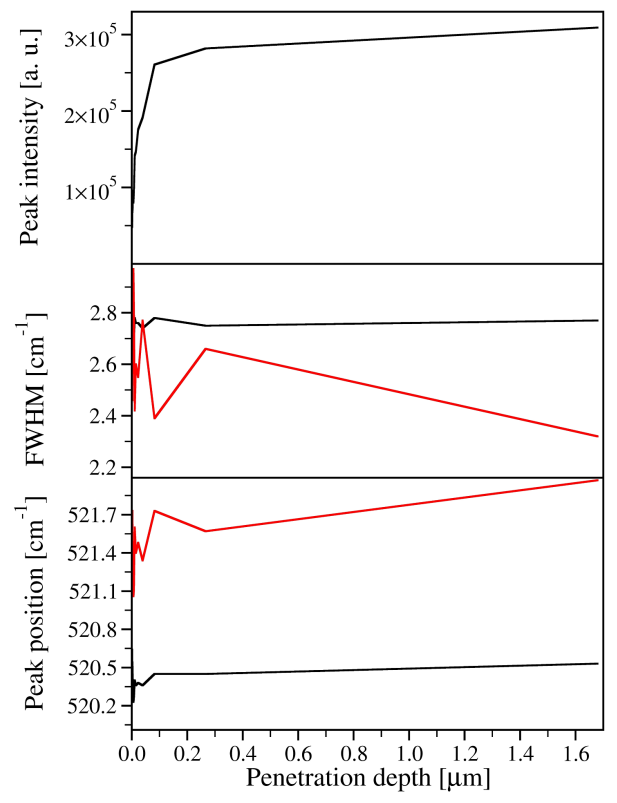

Fig. 4. Analysis of $z$-scan Raman spectra with two Lorentzian profiles (silicon peak). The following frames (starting from bottom) present: maximum of Lorentzian profiles, FWHM of the profiles and intensity of peak 1 .
The other problems coming out by analysis of Raman signals from $\mathrm{SiO}_{2}$ layer. Since the signal-to-noise ratio is small the precise analysis of the data requests first denoising of measured data. The comparison between wavelet analysis and fast Fourier transform (FFT) shows that wavelet gives much better results in comparison with FFT. The detailed discussion of the denoising procedure and its influence on the spectra will be presented in subsequent work.

\section{Conclusions}

The addition of about $1 \% \mathrm{Cu}$ to the metallic contact allows to obtain very strong signal from the part of MOS structure placed under the gate. The possible explanation of the magnification of the Raman signal observed from this area is surface enhanced raman scattering. The detailed investigations of the mechanism of amplification are necessary to establish the nature of the phenomenon.

The mechanical stress existing in MOS structure is depth dependent phenomenon. It is clearly seen by the Raman measurements with changing effective penetration depth. The mathematical model should contain two Lorentzian profiles in order to reproduce properly the shape of measured the Raman spectrum.

\section{Acknowledgments}

The authors thanks Mr. Mariusz Latek for his great help by realization of the experimental part of this work.

\section{References}

[1] I. De Wolf, J. Vanhellenmont, A. Romano-Rodriguez, H. Norström, H.E. Maes, J. Appl. Phys. 71, 898 (1992).

[2] B. Dietrich, V. Bukalo, A. Fischer, K.F. Dombrowski, E. Bugiel, B. Kuck, H.H. Richter, Appl. Phys. Lett. 82, 1176 (2003).

[3] G.H. Loechelt, N.G. Cave, J. Menéndez, J. Appl. Phys. 86, 6164 (1999).

[4] S.J. Harris, A.E. O'Neill, W. Yang, P. Gustafson, J. Boileau, W.H. Weber, B. Majmudar, S. Ghosh, J. Appl. Phys. 96, 7195 (2004).

[5] S. Narayanan, S. Kalidindi, L. Schadler, J. Appl. Phys. 82, 2595 (1997).

[6] J. Vanhellenmont, S. Amelinckx, C. Claeys, J. Appl. Phys. 61, 2170 (1987). 